Rev. Bras. Ciênc. Vet. 1(1):07-11, set. /dez. 1994

\title{
Enumeração e identificação de Vibrio parahaemolyticus em lulas frescas comercializadas no município de Niterói RJ Brasil
}

\author{
(Enumeration and identification of Vibrio parahaemolyticus in fresh squids \\ commercialized at Niterói, Rio de Janeiro State, Brazil)
}

\author{
LIMA, Francisco Carlos de*, OLIVEIRA, Luiz Antônio Trindade de ${ }^{\star \star}$ e MANO, Sergio Borges ${ }^{\star \star \star}$
}

\begin{abstract}
Resumo
Com o objetivo de verificar as condições higiênico-sanitárias do pescado comercializado no município de Niterói, efetuou-se um estudo sobre a enumeração e identificação de Vibrio parahaemolyticus em lulas frescas. Foram utilizadas 50 amostras de lulas frescas, identificadas como pertencentes à espécie Dorytheutis brasiliensis Blainville, 1823. A identificação e enumeração do $V$. parahaemolyticus foram baseadas nos métodos descritos pela International Commission on Microbiological Specifications for Foods ICMSF (1983). A classificação de Heiberg (1936) foi utilizada como teste complementar. O V. parahaemolyticus foi identificado em três (6\%) amostras, sendo que, em uma o vibrio se desenvolveu em meio com concentração salina a 10\%, na prova de halofilismo. O V: parahaemolyticus apresentou os NMPs (Número Mais Provável) com média de 19,3.bactérias/g. As três amostras de V. parahaemolyticus submetidas à classificação de Heiberg apresentaram perfil compatível com o grupo VII (Sacarose -, Manose + e Arabinose +). Concluiu-se que, mesmo sob comercialização inadequada, com manipulação e resfriamento precários, as amostras analisadas apresentaram o V. parahaemolyticus com freqüências e NMPs baixos.
\end{abstract}

Palavras-chave: Vibrios marinhos, Vibrio parahaemolyticus

\section{Introdução}

O primeiro isolamento do Vibrio parahaemolyticus foi efetuado por Fungino et al., em 1951, no Japão, em material de necrópsia de um surto de gastrenterite que envolveu 272 pessoas, com 20 óbitos. Este agente foi também isolado de amostras de "shirasu" (sardinhas jovens semi- dessecadas e cozidas), considerado o veículo da toxinfecção.

O V. parahaemolyticus é uma bactéria Gram negativa, em forma de bastão curto, exibindo pleomorfismo, em que são freqüentes as formas: curvas e cocóides (Bartley e Slanetz, 1971; Vanderzant e Nickelson, 1972; Nelson e Potter, 1976). É móvel, apresentando somente flagelo polar quando em meio líquido. É anaeróbio facultativo (Fishbein e Wentz, 1973; Nelson e Potter, 1976), mostrando tendência à coloração bipolar (Wanderzant e Nickelson, 1972), e desenvolvendo-se melhor em $\mathrm{pH}$ alcalino entre 7,5 e 8,5 e temperaturas entre 35 e $37^{\circ} \mathrm{C}$ (Sakazaki et al., 1963). É um microrganismo halofílico, necessitando para o seu isolamento e identificação o mínimo de um por cento de. $\mathrm{NaCl}$ (Johnson et al., 1971). Distribui-se amplamente no ambiente marinho, principalmente em águas costeiras e estuarinas temperadas (Covert e Woodburn, 1972; Thompson et al., 1976; Kaysner, 1981; Shinoda et al., 1983).

No Japão, de todas as possíveis causas de surtos de gastrenterites, o V. parahaemolyticus tem sido responsabilizado pela maioria dos casos (Sakazaki et al., 1963; Fishbein e Wentz, 1973). Somente durante o período de 1952 a 1955, ocorreram neste país, segundo Kawabata et al. (1957), 866 surtos de toxinfecções alimentares envolvendo aproximadamente 5.000 pessoas. Destas, 758 tinham como veículo a ingestão de cefalópodes. As características epidemiológicas, na maioria dos casos, apontavam o V. parahaemolyticus como agente nosológico. Zen-Yoji et al. (1965), constataram que, de 2.073 pacientes com toxinfecções alimentares, $606(29,2 \%)$ eram devido ao $\mathrm{V}$. parahaemolyticus.

* Professor Adjunto N

** Professor Títular

*** Professor Assistente 1

Departamento de Tecnologia dos Alimentos da Faculdade de Veterinária da Universidade Federal Fluminense - UFF. 
A presença e o desenvolvimento sazonal do $\mathrm{V}$. parahaemolyticus em água e pescado marinhos são sempre um risco potencial para a Saúde Pública. Quantidades relativamente baixas, em torno de 11.000 bactérias/g de amostra, podem dar início à toxinfecção (Thompson Jr. et al., 1976).

Esta pesquisa tem por objetivo a enumeração e indentificação do V. parahaemolyticus, para maior conhecimento das características microbiológicas relacionadas com o estado higiênico-sanitário do pescado comercializado no município de Niterói (Rio de Janeiro)

\section{Material e Métodos}

No experimento foram utilizadas 50 amóstras de lulas frescas, obtidas no mercado varejista do município de Niterói-RJ, no período de março a dezembro de 1988. Estas eram colocadas em sacos plásticos pelos próprios vendedores, com a finalidade de retratar as características reais do produto oferecido ao consumidor, e imediatamente levadas em isopor com gelo para o Laboratório de Controle Microbiológico de Produtos de Origem Animal da Faculdade de Veterinária da Universidade Federal Fluminense (UFF). Com os cuidados normais de assepsia, as partes comestíveis (manto e tentáculos) eram fragmentadas e, a seguir, homogeneizadas em liquidificador, por dois minutos, em rotação média.

Para a enumeração e identificação do V. parahaemolyticus, tomou-se como base a metodologia prescrita pela International Commission on Microbiological Specifications for Foods (ICMSF) de 1983, com algumas modificações, descritas a seguir:

a) Usou-se para o Número Mais Próvavel (NMP) o meio GSTB (Akiyama e Takizawa, 1963, segundo Barros, 1977) ao invés de caldo polimixina.

b) Na prova de Voges-Proskauer foi usada a temperatura de $35-37^{\circ} \mathrm{C} / 24-48$ horas, segundo MacFaddin (1985), ao invés de $27-29^{\circ} / 18$ horas.

c) Na formulação do GSTB usou-se o Tween 80 (Merck. Art. 822187) em substituição ao teepol. Ambas as substâncias têm a função de aumentar a tensão superficial do meio, dificultando a motilidade, o que evita a formação do "swarming" ou espalhamento de determinados grupos de vibrios, conferindo-se com isso maior número de Unidades Formadoras de Colônias (UFC) isoladas.

Como teste auxiliar para a identificação do $\mathrm{V}$. parahaemolyticus utilizou-se a classificação de Heiberg (1936), que é baseada nas respostas à fermentação dos açúcares manose, sacarose e arabinose. Por esta classificação o V. parahaemolyticus pode pertencer a três grupos distintos: o V (manose +/ sacarose -/ arabinose -); o VII (manose + / sacarose - / arabinose +); e o VIII (manose - / sacarose -/ arabinose +) (Chatterjee, 1974)

\section{Resultados}

A pesquisa mostrou que as 50 amostras inoculadas em meio GSTB apresentaram crescimento. Quando inoculados em agar TCBS (Thiossulfate-citrate-biliar salts), em 28 (56\%)
Tabela I - Enumeração de víbrios totais e $V$. parahaemolyticus em amostras de lulas.

\begin{tabular}{ccc}
\hline No & $\begin{array}{c}\text { Vibrios Totais } \\
\text { NMP bact./g }\end{array}$ & $\begin{array}{c}\text { Vibrio parahaemolyticus } \\
\text { NMP bact./g }\end{array}$ \\
\hline 01 & 15 & - \\
02 & 4 & - \\
03 & 15 & - \\
04 & 93 & - \\
05 & 43 & 43 \\
06 & 23 & - \\
07 & 460 & - \\
08 & 15 & - \\
09 & 4 & - \\
10 & 4 & - \\
11 & 4 & - \\
12 & 11 & - \\
13 & 3 & - \\
14 & 23 & - \\
15 & 7 & - \\
16 & 150 & 4 \\
17 & 21 & - \\
18 & 15 & 11 \\
19 & 93 & - \\
20 & 11 & - \\
21 & 21 & - \\
22 & 75 & - \\
23 & 460 & - \\
24 & 39 & - \\
25 & 9 & - \\
26 & 1.100 & \\
27 & 28 & \\
28 & $>2.400$ & \\
& & - \\
\hline & & - \\
\hline
\end{tabular}

Tabela II - Perfil bioquímico do V. parahaemolyticus, isolado de amostras de lulas.

Provas

V. parahaemolyticus

\begin{tabular}{|c|c|c|}
\hline \multirow{2}{*}{\multicolumn{2}{|c|}{$\begin{array}{l}\text { Citocromo oxidase } \\
\text { VP }\end{array}$}} & + \\
\hline & & \\
\hline \multicolumn{2}{|c|}{ Crescimento a $42^{\circ} \mathrm{C}$} & + \\
\hline \multicolumn{2}{|c|}{ Lisina } & + \\
\hline \multirow{3}{*}{$\begin{array}{l}\text { Arginina } \\
\text { Halofilismo }\end{array}$} & & - \\
\hline & $0 \%$ & - \\
\hline & $6 \%$ & + \\
\hline & $8 \%$ & + \\
\hline & $10 \%$ & $1+/ 2-$ \\
\hline \multicolumn{2}{|l|}{ Glicose } & + \\
\hline \multicolumn{2}{|l|}{ Sacarose } & - \\
\hline \multicolumn{2}{|l|}{ Manitol } & + \\
\hline
\end{tabular}

$(+)=$ amostra positiva

$(-)=$ amostra negativa

amostras evidenciou-se a formação de UFCs sacarose positivas, de tamanhos variados, com tendência a grandes, e destas, em três (6\%), foi verificado o desenvolvimento concomitante de UFCs verde-azuladas, pequenas, caracterís- 


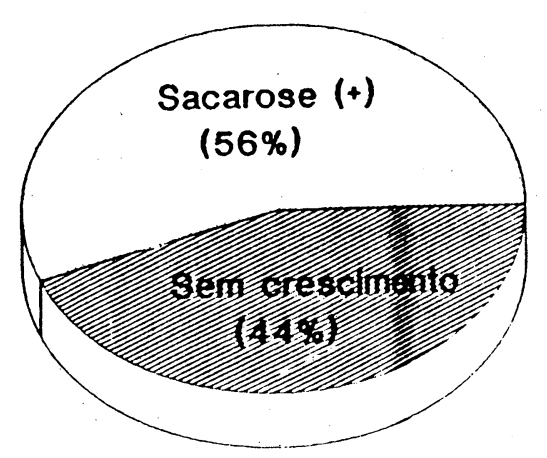

Gráfico 1 - Percentual de freqüência de bacérias sacarose positivas em 50 amostras de lulas

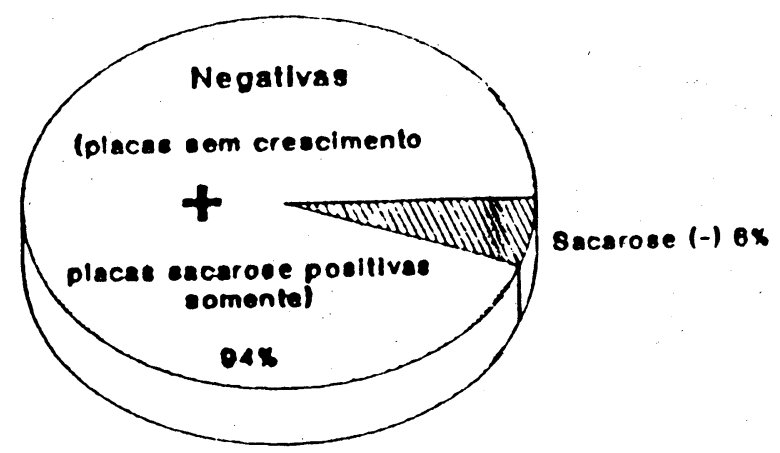

Grífico 2 - Percentual de treqüência de bactérias sacarose negativas em 50 amostras de lulas

ticas do V. parahaemolyticus (Gráficos 1 e 2). O maior número de UFCs verdes encontradas foi de quatro por placa.

O exame morfológico evidenciou que todas as UFCs (verdes e amarelas) eram formadas por bactérias Gram negativas, pleomórficas, porém com número significativo de organismos em forma de bastões curvos.

Excluindo-se do somatório a amostra com NMP > 2.400/ g (Tabela I), a média dos NMPs foi de 86,5 bactérias/g e, com $20(71,4 \%)$ destas amostras apresentando seus NMPs abaixo do índice médio

As bactérias oriundas das UFCs verde-azuladas apresentaram-se móveis no agar motilidade e cresceram meIhor em caldo tripticase soja que no agar correspondente. Nas semeaduras em TSI, produziram acidificação da base e alcalinização do bisel, sem formação de gás ou $\mathrm{H}_{2} \mathrm{~S}$ :

Os testes bioquímicos evidenciaram uma grande semeIhança com o perfil bioquímico do Vibrio parahaemolyticus (Tabela II); embora uma das amostras tenha se desenvolvido em concentração salina a 10\%, na prova do halofilismo.

As três amostras de $V$. parahaemolyticus submetidos à classificação de Heiberg, apresentaram perfil compatível com o grupo VII (sacarose -/ manose +/ arabinose +).

\section{Disculssão}

Em amostras de mercado, os níveis percentuais de isolamento do V. parahaemolyticus podem sofrer oscilações consideráveis, sobretudo em face do grau de resfriamento aplicado. Kampelmacher et al., (1970) observaram um nível de contaminação, pelo $\mathrm{V}$. parahaemolyticus, de somente 0,3\% em peixes e bivalves nos mercados da Holanda, enquanto Bockemuhl e Triemer (1974) reportaram em Lomé (África), um índice de positividade de $44,2 \%$. Em peixes capturados em alto mar, o índice chegava a 14,3\% (após estar com apenas 0,5\% no momento da captura), e 67,3\% em pescado de águas costeiras, que já apresentava percentuais bastante altos na captura (em torno de 47\%).

Não sendo filtradoras, não possuindo hábitos bentônicos e tendo predileção por águas não poluídas, as lulas teriam comportamento semelhante aos peixes de alto mar, no que concerne à quantidade de vibrios presentes em suas superfícies comporais. O percentual de isolamento $(6 \%)$ do $\mathrm{V}$. parahaemolyticus encontrado no presente trabalho, pode ser considerado satisfatório, em vista da manipulaçãlo e resfriamento inadequados recebidos pelas lulas, quando expostas à venda. As amostras de Kampelmacher et al. (1970), além de originárias do Mar do Norte, de águas frias, eram mantidas resfriadas, dificultando com isso a multiplicação do vibrio.

A relação existente entre o número de isolamentos de vibrios sacarose positivos e o V. parahaemolyticus mostrou que a presença dos primeiros era muito mais efetiva. Segundo Zen-Yoji et al. (1965), em peixes e bivalves, a diferença na quantidade de isolamentos de vibrios sacarose positivos, chegava a quase dez vezes o número de isolados do $\checkmark$. parahaemolyticus (90,2 e 9,8\% respectivamente). A relação aproximada de 10:1 foi também observada em lulas. As amostras sacarose positivas apresentaram um percentual de isolamento de $56 \%$ contra os $6 \%$ do $\mathrm{V}$. parahaemolyticus.

Em 1972, Kampelmacher e Jansen afirmavam, que devido as discrepâncias de natureza intrínseca, alguns testes bioquímicos apresentavam respostas que poderiam resultar na agrupagem imprecisa dos organismos da família Vibrionaceae. O próprio caráter halofílico do $\mathrm{V}$. parahaemolyticus, já que seu crescimento pode apresentar variações em diferentes concentrações salinas, não deveria ser usada, segundo Twedt et al. (1969) e Vanderzant e Nickelson (1972), como elemento chave na sua identificação, embora vários autores citem o organismo como medianamente halofílico, não se desenvolvendo em meios com 10\% de $\mathrm{NaCl}$ (Sakazaki et al., 1963; Jegathsan e Paramasivan, 1976; Pezzlo et al., 1979; Blake et al., 1980). No entanto, algumas citações apontam amostras do $\mathrm{V}$. parahaemolyticus ocasionalmente positivas em cultivos salinos a 10\% (Baross e Liston, 1968; Kampelmacher e Jansen, 1972; Kristensen, 1974; Chun et al., 1974); enquanto outras indicam números bem mais elevados como a de Zen-Yoji et al. (1965), que verificaram 15 amostras positivas em 52 (26,3\%) isolados; de Gjerde e Boe (1981), com 10 positivas em 15 (66,6\%); de Colwell (1970), em, até mesmo, 100\% das amostras testadas.

No presente trabalho, uma das amostras identificadas com V. parahaemolyticus cresceu em caldo triptona com $10 \%$ de $\mathrm{NaCl}$, apesar deste crescimento não ser tão profuso quanto os observados nos meios de concentração de 6 a $8 \%$. Embora as características bioquímicas desta amostra 
estejam em concordância com alguns autores citados, o número reduzido đe isolamentos do V. parahaemolyticus não póssibilitou o aprofundamento das observações, ou seja, se no caso de maior número de isolamentos ter-se-ia a oportunidade do encontro de outras amostras com as mesmas características. Concluiu-se, sobre este aspecto, que as provas bioquímicas inerentes à um determinado grupo não devem ser analisadas separadamente e sim em conjunto, apontando ainda a prova do halofilismo a 10\% como de grande importância auxiliar na identificação do $\mathrm{V}$. parahaemolyticus.

O teste de fermentação da arabinose, de acordo com Leitão e Arina (1975), é de grande valor na caracterização do V. parahaemolyticus, sendo utilizado neste trabalho como prova integrante na classificação de Heiberg. As 3 amostras isoladas mostraram-se positivas à fermentação de arabinose como as descritas por Chatterjee et al. (1980), Kampelmacher e Jansen (1972), Olsen et al. (1978) e Blake et al. (1980) indicando serem pertencentes ao grupo VII de Heiberg.

\section{Summary}

\section{Enumeration and identification of Vibrio parahaemolyticus in fresh squids commercialized at Niterói Rio de Janeiro State, Brazil}

A study on the enumeration and identification of Vibrio parahaemolyticus in fresh squids in Niterói was undertook aiming at checking the hygienic-sanitary conditions of fish commercialized in that city; . Fifty fresh squids (Dorytheutis brasiliensis, Blainville, 1823). Methods described by the International Comission on Microbiological Specification for Foods-ICMSF (1983) were used to identify and enumerate $V$. parahaemolyticus and Heiberg's classification was a complemental test. $V$. parahaemolyticus was identified in three samples (6\%); in one of them it grew in an environment with $10 \%$ salt concentration during halophilism probe. Most probable number (MPN) average for $V$. parahaemolyticus was 19,3 bacteria per gram. The three samples submitted to Heiberg's classification showed a profile compatible with group VII (sacarose-, manose+, arabinose-). We concluded that, even under inadequate commercialization, manipulation and refrigeration conditions, $V$. parahaemolyticus frequencies and MPNs are low.

Key words: Marine vibrios, Vibrio parahaemolyticus

\section{Referências Bibliográficas}

BAROSS, J., LISTON, J. Isolation of Vibrio parahaemolyticus from the Northwest Pacific. Nature, Lon., v. 217, p. 1263-1264, 1968.

BARROS, G.C. Vibrio parahaemolyticus: Isolamento e Identificação em Crustáceos e Moluscos da Baía de Sepetiba. Niterói, 1977. 70p. Dissertação de Mestrado em Medicina Veterinária (Área de Concentração de Higiene Veterinária e Processamento Tecnológico de Produtos de Origem Animal). Universidade Federal Fluminense.

BARTLEY, C.H.; SLANETZ, L.W. Occurrence of Vibrio parahaemolyticus in Estuarine Waters and Oysters of New Hampshire. Applied Microbiology, v. 107, n. 1, p. 268-294, 1971.
BLAKE, P.A., WEAVER, R.E., HOLLIS, D.G. Diseases of Humans (other than cholera) Caused by Vibrios. Annual Review of Microbiology, v. 34, p. 341-367, 1980.

BOCKEMUHL, J., TRIEMER, A. Ecology and Epidemiology of Vibrio parahaemolyticus on the Coast of Togo. Bulletin of the World Health Organization, v. 51, n. 4, p. 353-360, 1974.

CHATTERJEE, B.D. Present Status of:Heiberg Groups for classifying Choleralike Organisms. Indian Journal of Medical Research, v. 2. p. 479-483, 1974.

CHATTERJEE, B.D., GORBACH, S.L. NEOGY, K.N. Characteristics of NonCholera Vibrios Isolated from Patients with Diarrhoea, Journal of Medical Microbiology, V. 3, n.4, p. 677-682, 1970.

CHUN, D., CHUNG, J.K., SEOL, S.Y. TAK, R. Vivrio parahaemolyticus in the Republic of Korea. American Journal of Tropical Medicine and Hygiene, v. 23, n.6, p. 1125-1130, 1974.

COLWELL, R.R. Polyphasic Taxonomy of the Genus Vibrio: Numerical Taxonomy of Vibrio cholerae, Vibrio parahaemolyticus, and Related Vibrio Species. Journal of Bacteriology, v. 104, n. 1, p. 410-433, 1970.

COVERT, D., WOODBURN, M. Relationships of Temperature and Sodium Chloride Concentration to the Survival of Vibrio parahaemolyticus in Broth and Fish Homogenate. Applied Microbiology. v. 23, n. 2, p. 321-325, 1972.

FISBBEIN, M., WENTZ, B. Vibrio parahaemolyticus Methodology for Isolation from Seafoods and Epidemic Specimens. Journal of Milk and Food Technology v. 36, n. 2, p. 118-123, 1973.

FUJINO, T., OKUNO, Y., NAKADA, D., AOYAMA, A., FUKAI, K., MURAl, K., UEHO, T. On the Bacteriological Examination of Shirasu Food Poisoning. Journal of the Japanese Association of Infectious Diseases, v. 25, p. 11 $16,1951$.

GJERDE, J., BOE, B. Isolation and Characterization of Vibrio alginolyticus and Vibrio parahaemolyticus from the Norwegian Coastal Environment. Acta Veterinaria Scandinavica, v. 22, p. 331-343, 1981.

HEIBERG, B. The Biochemical Reactions of Vibrios. Journal of Hygiene, Lond. v. 36, p. 114-117, 1936.

International Comission on Microbiological Specifications for foods. Microorganismos de los alimentos. 2 ed. Zaragoza: Acribia, 1983. 2 v. v.1: Tecnicas de analises microbioligico. 431p.

JEGATHESAN, M., PARAMASIVAM, T. Emergence of Vibrio parahaemolyticus as an Important Cause of Diarrhea in Malaysia. American Journal of Tropical Medicine and Hygiene, v. 25, n.1, p. 201-202, 1976.

JOHNSON, H.C., BAROSS, J.A., LISTON, J. Vibrio parahaemolyticus and its Importance in Seafood Hygiene. Journal of the American Veterinary Medical Association, v. 159, n. 11, p. 1470-1473, 1971.

KAMPELMACHER, E.H., MOSSEL, D.A.A., JANSEN, L.M. van N., VICENTIE, $H$. A Survey of the Occurrence of Vibrio parahaemolyticus and $V$. alginolyticus, on Mussels and Oysters and in Estuarine Waters in the Netherlands. Journal of Applied Bacteriology, v. 35, n: 3, p. 431-438, 1972.

KAMPELMACHER, E.H., MOSSEL, D.A.A., JANSEN, L.M. van N., VICENTIE, $H$. A Survey on the Occurrence of Vibrio parahaemolyticus on Fish and Shellfish, Marketed in the Netherlands. Journal of Hygiene, Camb., v. 68, n. 2, p. 189-196, 1970.

KAWABATA, T., HALSTEAD, B.W., Judefind, T.F. A Report of a Series of Recent Outbreaks of Unusual Cephalopod and Fish Intoxications in Japan. American Journal of Tropical Medicine and Hygiene, v. 6, n. 5, p. 935939, 1957.

KAYSNER, C.A. Incidence of Vibrio alginolyticus and Bacteria of Sanitary Significance in the Bering Sea. Applied and Environmental Microbiology, v. 41 , n. 5, p. 1279-1282, 1981.

KRISTENSEN, K.K. The Ocurrence of Vibrio parahaemolyticus and $V$. alginolyticus in the Sound. Nordisk Veterinaer-Medicine, v. 26 n. 3/4, p. 188-196, 1974.

LEITÃO, M.F.F., ARINA, H.K. Vibrio parahaemolyticus no Ambiente Marinho do Estado de São Paulo. Coletânia do Instituto de Tecnologia de Alimentos, v.6, p. 149-166, 1975. 
MACFADDIN, J.F. Pruebas Bioquimicas para la Identificacion de las Bacterias de Importancia Clínica. Buenos Aires, Panamericana, 1985. $301 p$.

NELSON, K.J., POTTER, N.N. Growth of Vibrio parahaemolyticus at Low Salt Levels and in Nonmarine Foods. Journal of Food Science, v. 41, n. 6, p. 1413-1417; 1976.

OLSEN, H. Vibrio parahaemolyticus Isolated from Discharge from the Ear in Two Patients Exposed to Sea Water. Acta Pathologica et Microbiologica Scandinavica. Section B: Microbiology et Immunology, v. 86, n. 4, p. 247-248, 1978.

PEZZLO, M., VALTER, P.J., BURNS, M.J. Wound Infection Associated With Vibrio alginolyticus. American Journal of Clinical Pathology, v. 71, n. 4, p. 476-478, 1979

SAKAZAKI, R., IWANAMI, S., FUKUMI, H. Studies on the Enteropatogenic, Facultatively Halophilic Bacteria, Vibrio parahaemolyticus. I. Morphological, Cultural and Biochemical Properties and its Taxonomical Positions. Japanese Journal of Medical Science and Biology, v. 16, p. $161-188,1963$.

SHINODA, S., NAKAHARA, N., NINIMIYA, Y., ITO, K., KANE, H., ZEN-YOTI, H., SAKAI, S., TERAYANA, T., KUDO, Y., ITO, T., BENOKI, M. NAGASAKI, M. Serological Method for Identification of Vibrio parahaemolyticus from
Marine Samples. Applied and Environmental Microbilogy, v. 45 n.1, p. 148-152, 1983.

THOMPSON, Jr. S.A. VANDERZANT, C., RAY, S.M. Effect of Processing, Distribution and Storage on Vibrio parahaemolyticus and Bacterial Counts of Oysters (Crassostrea virginica). Journal of Food Science, v. 41, n. 1, p. 123-127, 1976.

THOMPSON, S.A., VANDERZANT, C., RAY, S.M. Relationship of Vibrio parahaemolyticus in Oysters, Water and Sediment, and Bacteriological and Environmental Indices. Journal of Food Science v. 41, n. 1, p. 117 122, 1976

TWEDT, R.M., SPAULDING, P.L., HALL, H.E. Morphological, Cultural, Bochemical and Serological Comparison of Japanese Strains of Vibrio parahaemolyticus with Related Cultures Isolated in the United States. Journal of Bacteriology, V. 98, n. 2, p. 511-518, 1969.

VANDERZANT, C., NICKELSON, R. Procedure for Isolation and Enumeration of Vibrio parahaemolyticus. Applied Microbiology, v. 23, n.1, p. 26-33, 1972.

ZEN-YOJI, H., SAKAI, S., TERAYANA, T., KUTO, Y., ITO, T., BENOKI, M. NAGASAKI, M. Epidemiology, Enteropathogenicity, and Classification of Vibrio parahaemolyticus. Journal of Infectious Diseases, v. $115, n .5$, p. 436-444, 1965. 